\title{
Metis/Solar Orbiter Polarimetric Visible Light Channel Calibration
}

M.Casti*a, S. Fineschi a , G. Capobianco a , M. Romolib, E. Antonucci ${ }^{a}$, G. Nicolini ${ }^{a}$, G.

Naletto ${ }^{c}$, P. Nicolosid ${ }^{d}$, D. Spadaro ${ }^{e}$, V. Andrettaf, M. Castronuovo's, G. Massone a, R.

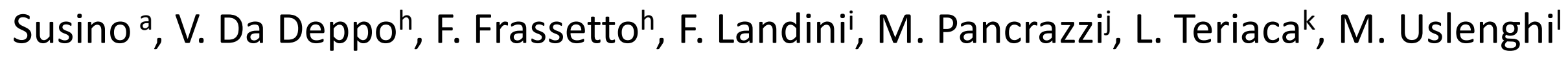




\section{Outline}

- Metis Coronagraph

- Polarization Modulation Package

- VL polarietric channel calibration

- Sun-disk imaging

- Polarization flat-fielding

- Results validation 


\section{Metis coronagraph}

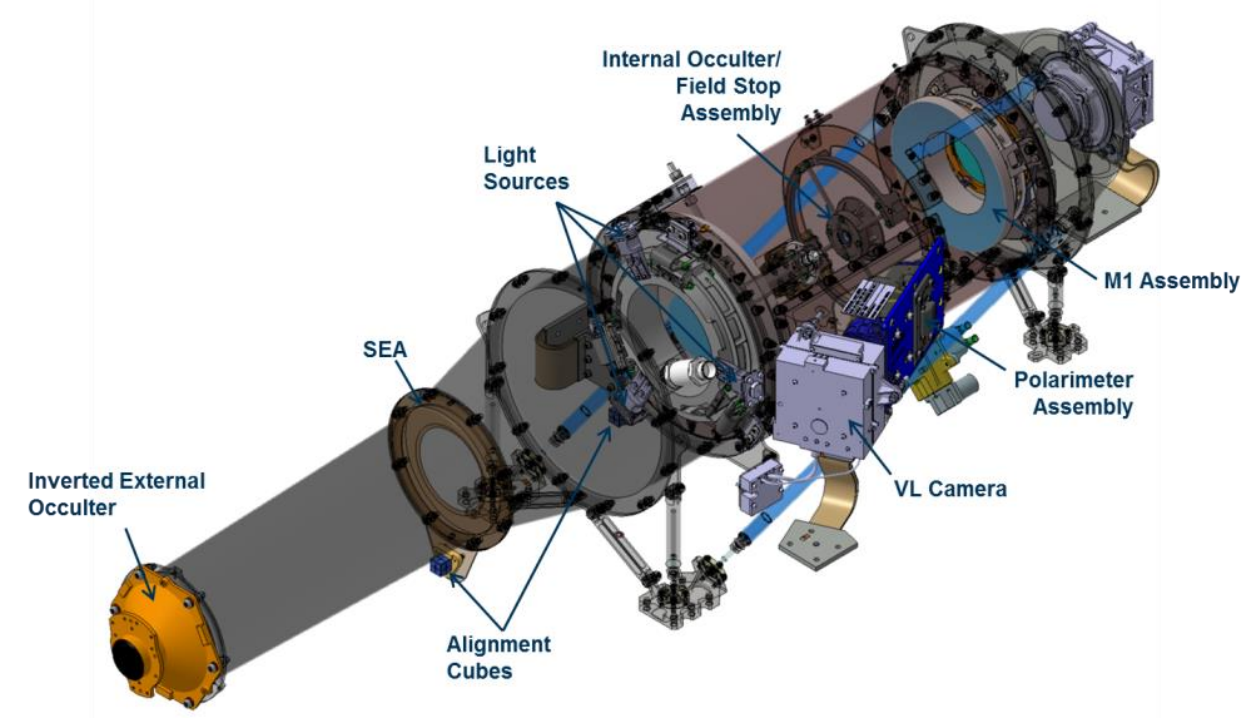

The Metis polarimeter includes:

- A quarter-wave retarder;

- a Polarization Modulation Package (PMP) composed of two Liquid Crystal Variable Retarder cells (LCVRs) $\rightarrow$ first use in space optical payload;

- A linear polarizer.
Metis will acquire simultaneous images of the full solar corona in:

- Linearly polarized visible-light $(580-640 \mathrm{~nm})$

- Narrow-band ultraviolet HI Lyman- $\alpha$ (121.6 nm)

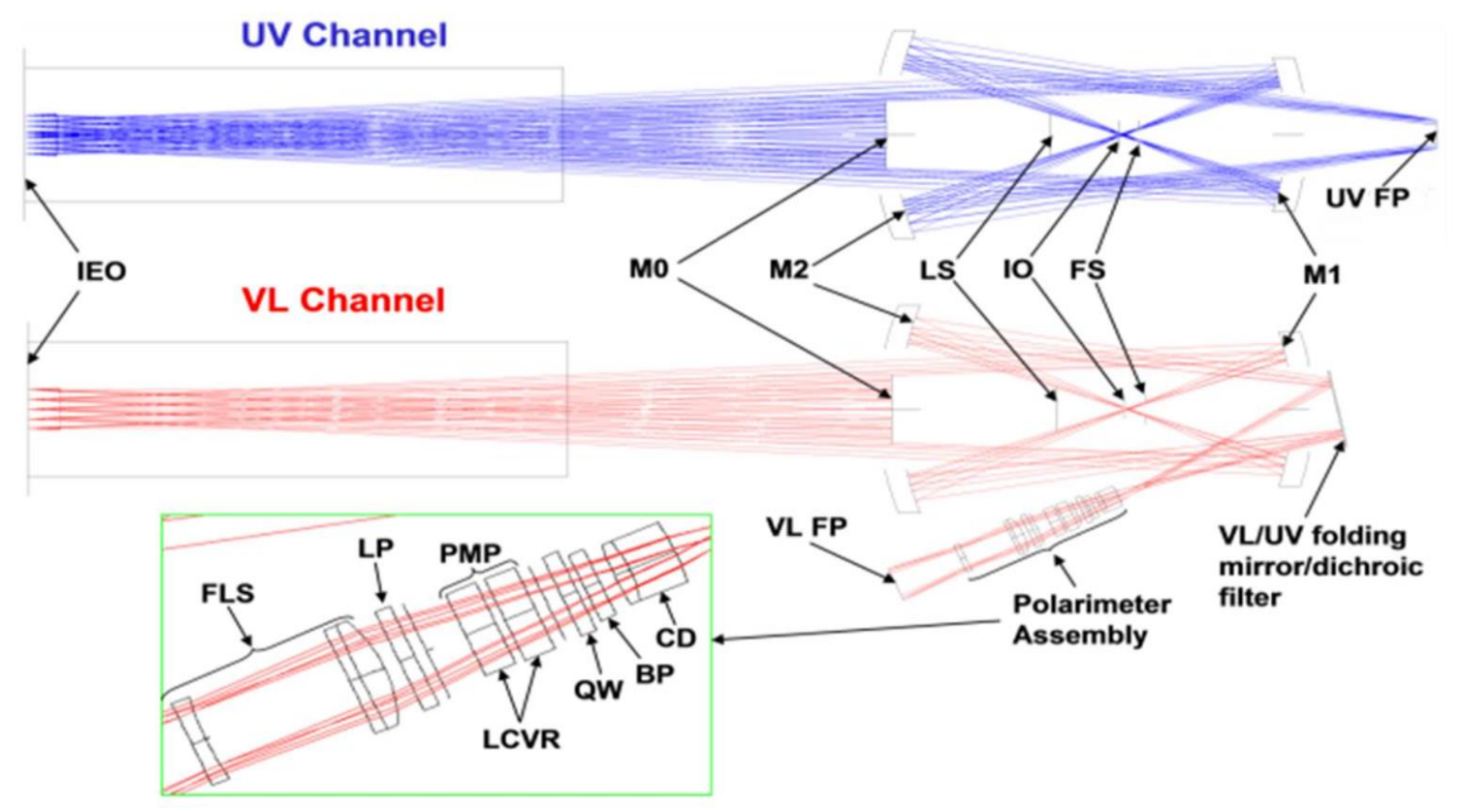




\section{Polarization Modulation Package (PMP)}

$\checkmark \quad$ The LCVRs consist of optically anisotropic liquid crystal molecules embedded between two glasses of $5 \mathrm{~mm}$ coated for one side with a transparent conductive material (indium tin oxide, ITO) and an alignment layer (rubbed polyimide) and the other side with an antireflective coating

$\checkmark \quad$ The effective birefringence of the cells can be changed by applying an electric field and rotating the crystal molecules.

$\checkmark$ A change in orientation of the molecules introduce an optical retardance in the orthogonal polarization components of the incoming light.

$\checkmark$ The calibration of the Metis Polarization and Modulation

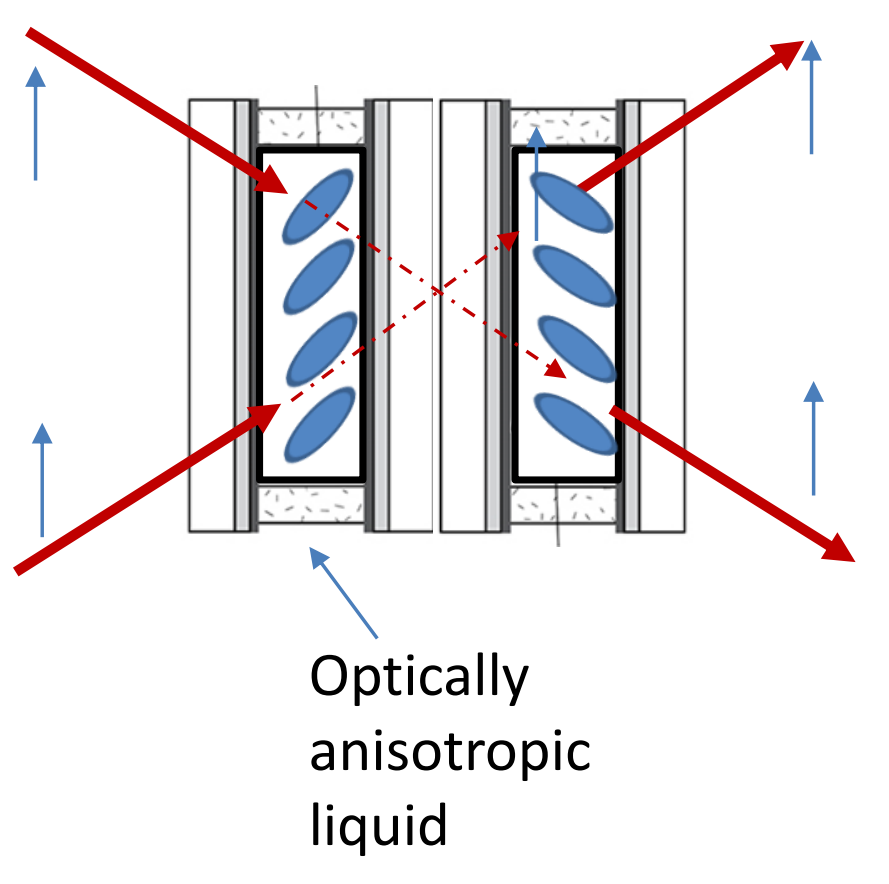
Package is mandatory for commanding the polarimeter during the observational sequences. 


\section{VL polarietric channel calibration}

Goals:

- Characterize the liquid crystals response to the applied voltage by retrieving the retardance-voltage curve;

- Characterize the polarimetric response in visible light on the overall instrument field of view by retrieving the pixel-by-pixel demodulation matrix

Test approach:

- a well-known polarized light was injected in the polarimeter and the LCVRs voltage was change to modulate the incoming light 


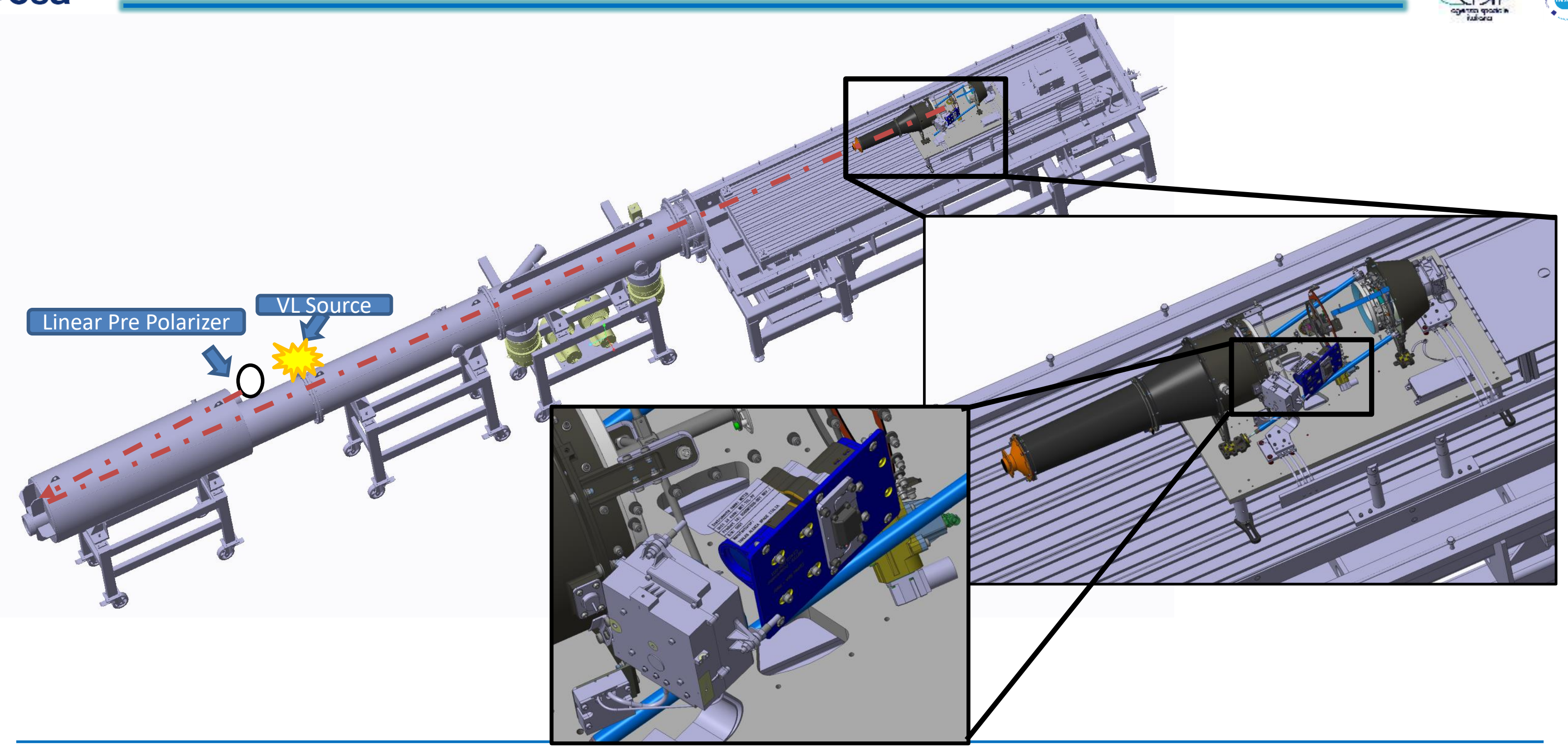




\section{Acquired data}
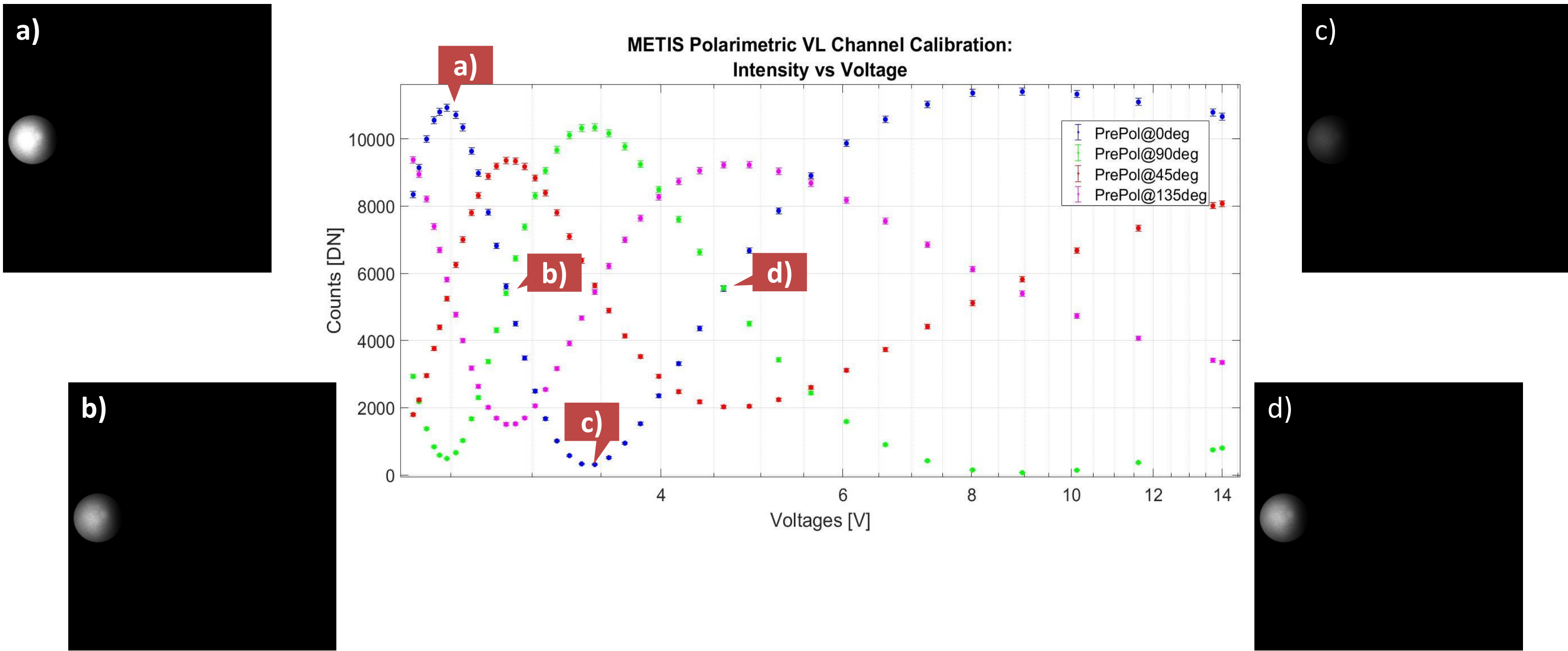

oltages [V]

d) 


\section{Data analysis}

Available data:

- $\quad$ Measured intensity $(I) \rightarrow$ mean of the DN within the area of interest

- $\quad$ Polarization degree $(p) \rightarrow$ verified measuring the light polarized by the linear prepolarizer (0.98)

- Linear Pre-Polarizer acceptance (transmission axis) angle $\rightarrow$ micrometric scale on the pre-polarizer

- $\quad$ Applied Voltage $\rightarrow$ telecommand

Angle of retardance introduced by the LCVR cells

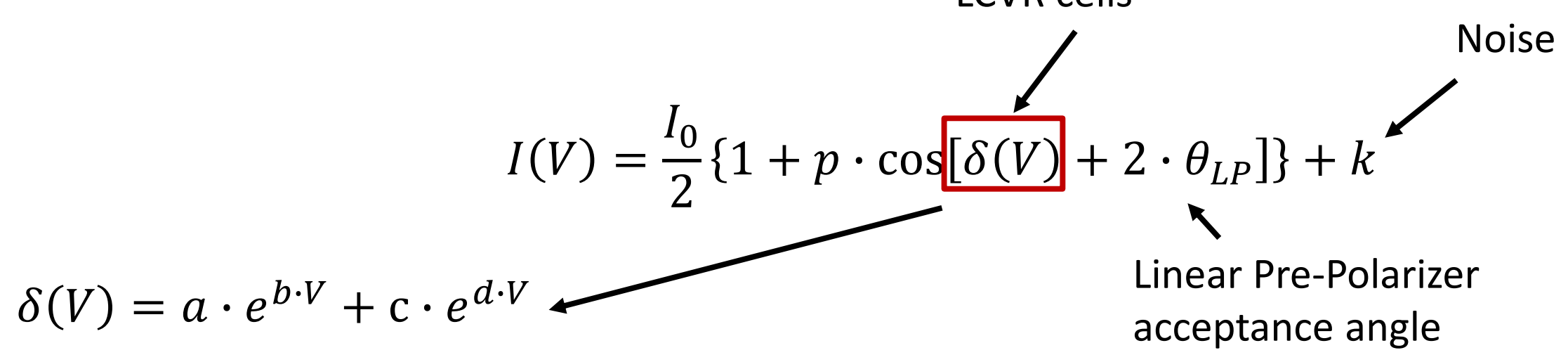




\section{Data Analysis Results}

Experimental Data

METIS Polarimetric VL Channel Calibration:

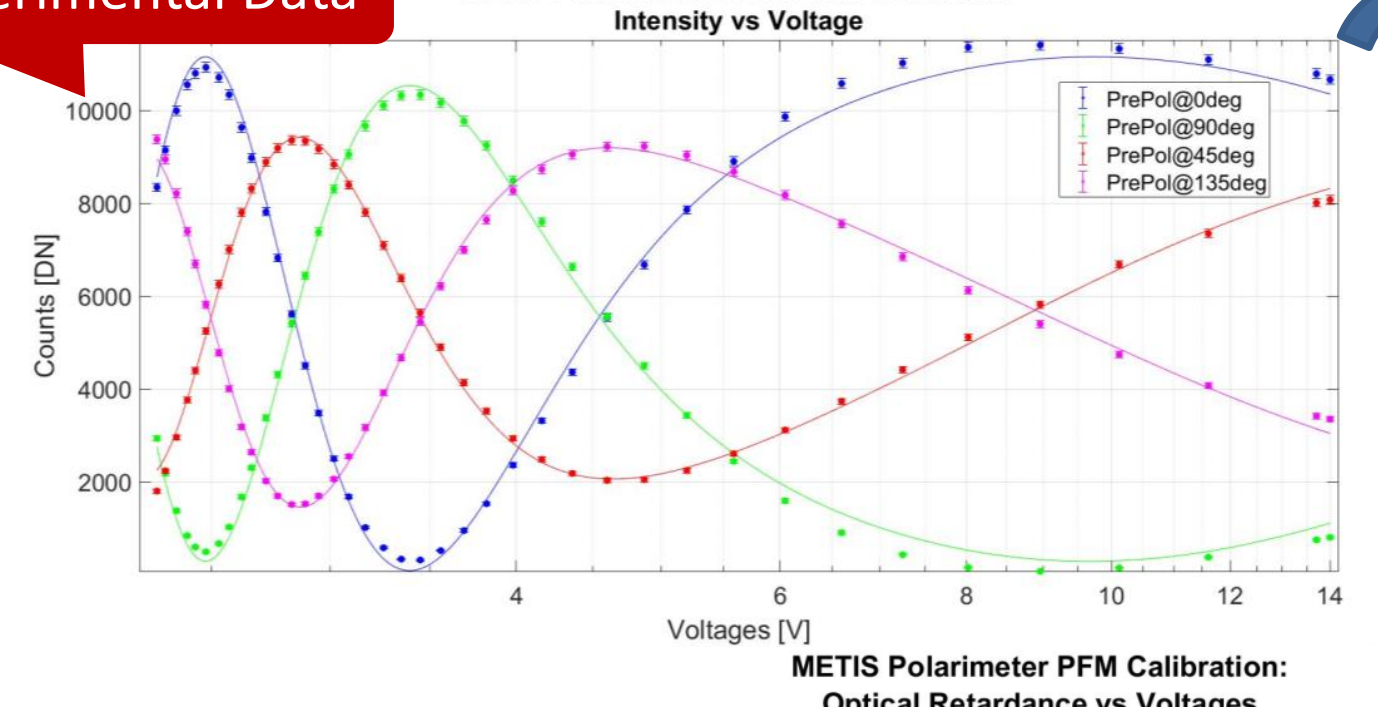

METIS Polarimeter PFM Calibration:
Optical Retardance vs Voltages

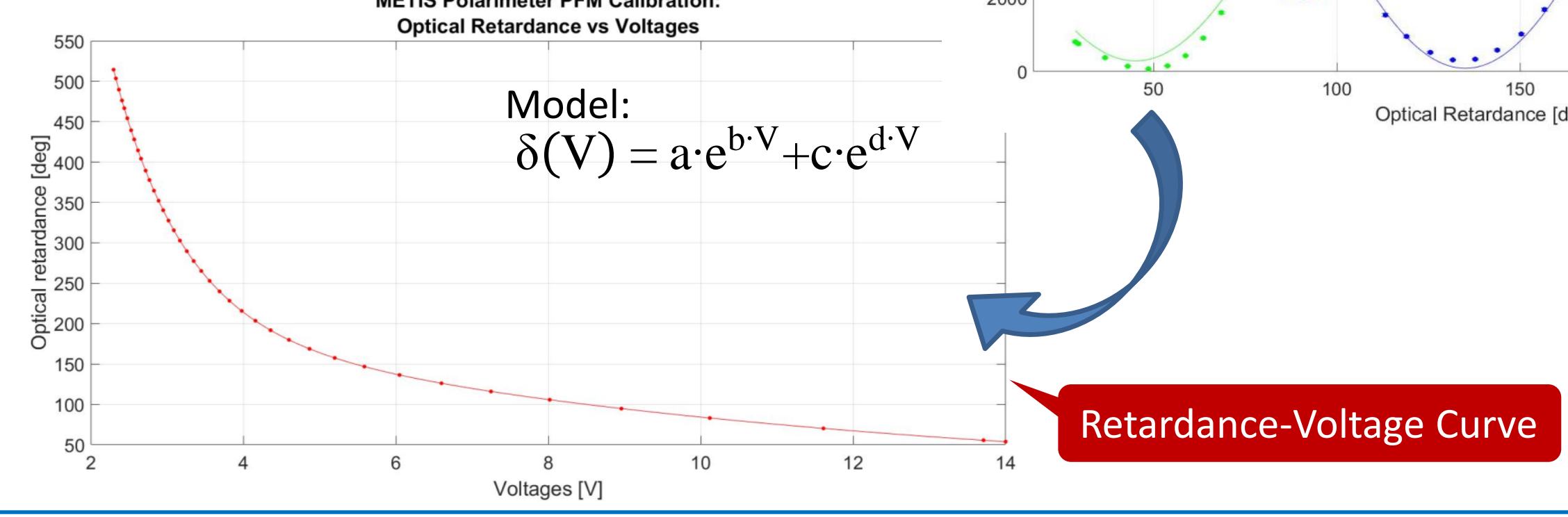

METIS Polarimeter PFM Calibration:

Malus Curve Malus curve

PrePol@0deg PrePol@45deg
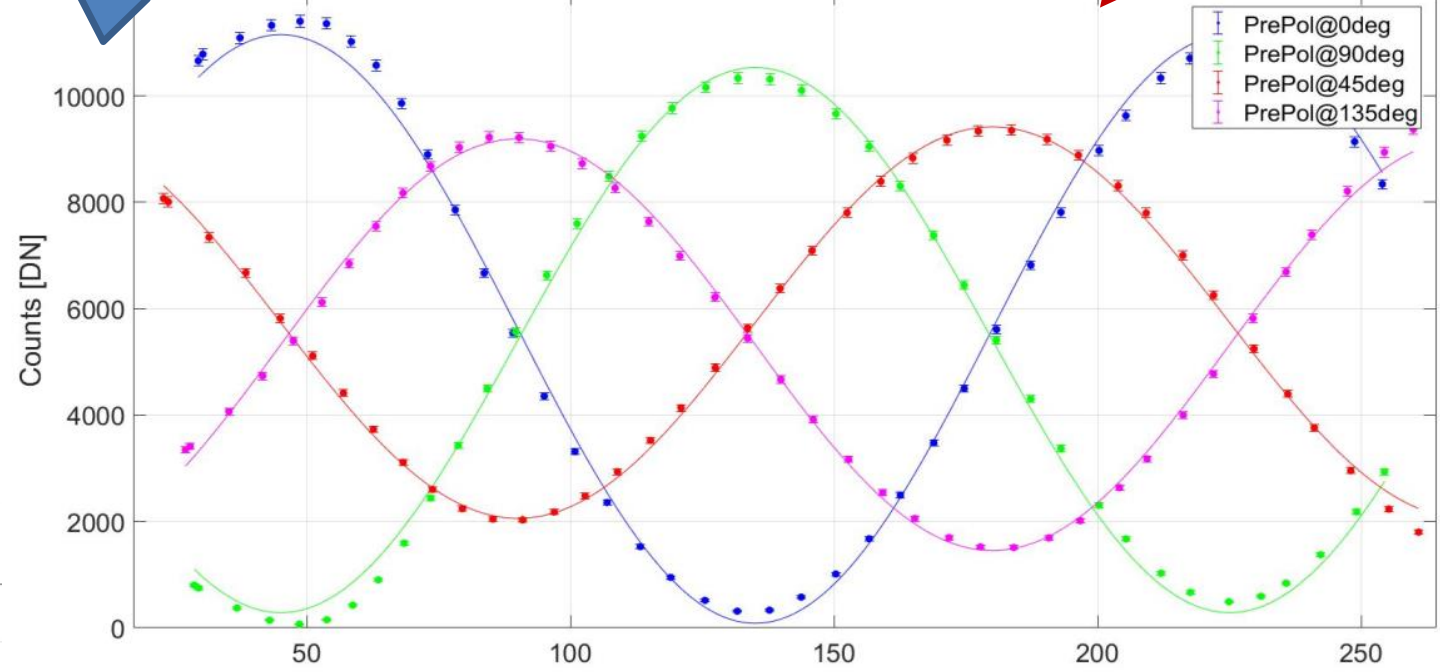

Model: 


\section{Polarimetric flat-fielding set-up}
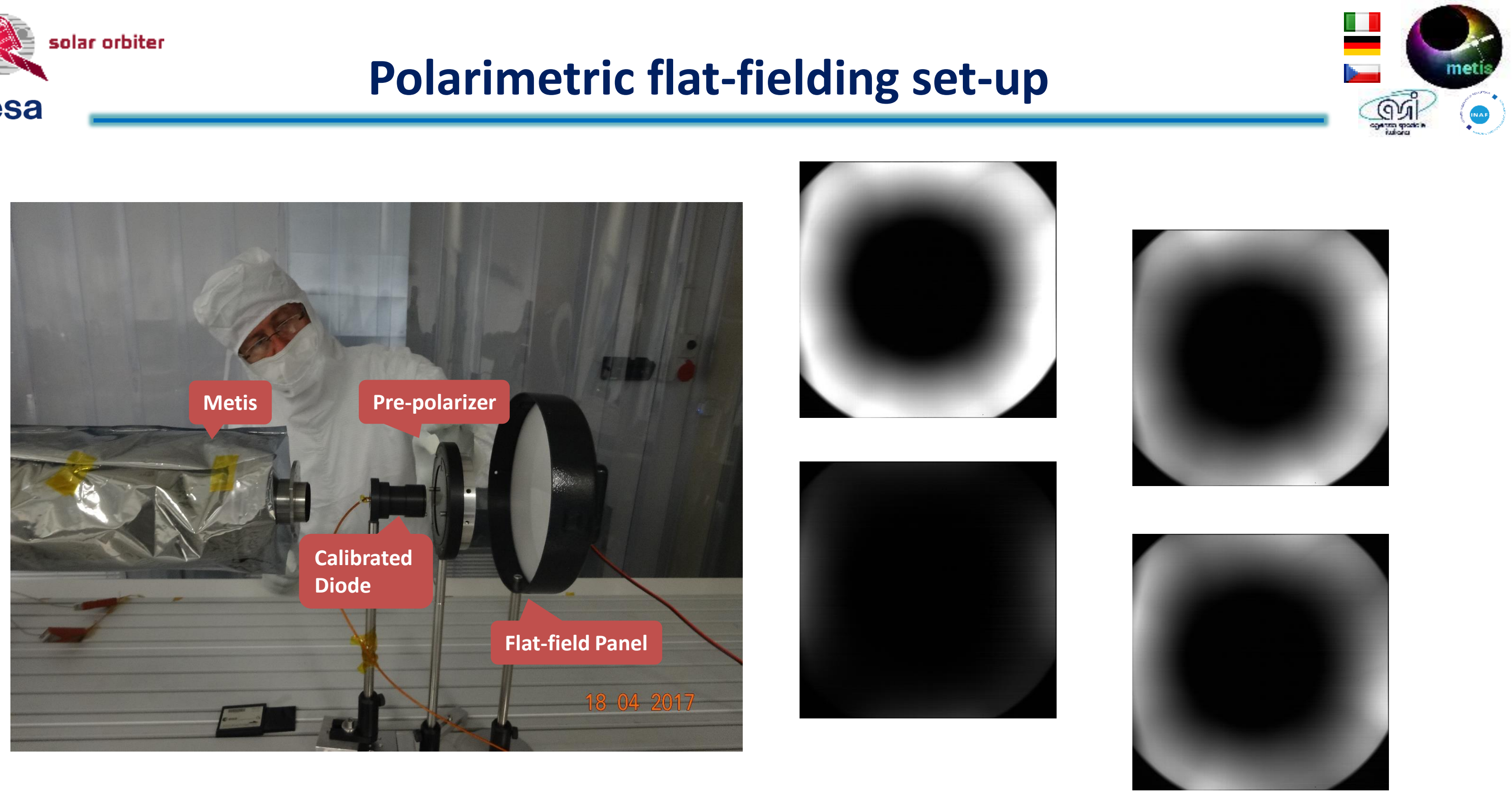


\section{Data Analysis}

Processing steps:

1. Retrive the coefficients of the modulation matrix for each pixel within the flat-field image

2. Retrieve the demodulation coefficients for each pixel by inverting the modulation matrix

3. Compute the efficiency vector

4. Verify the results

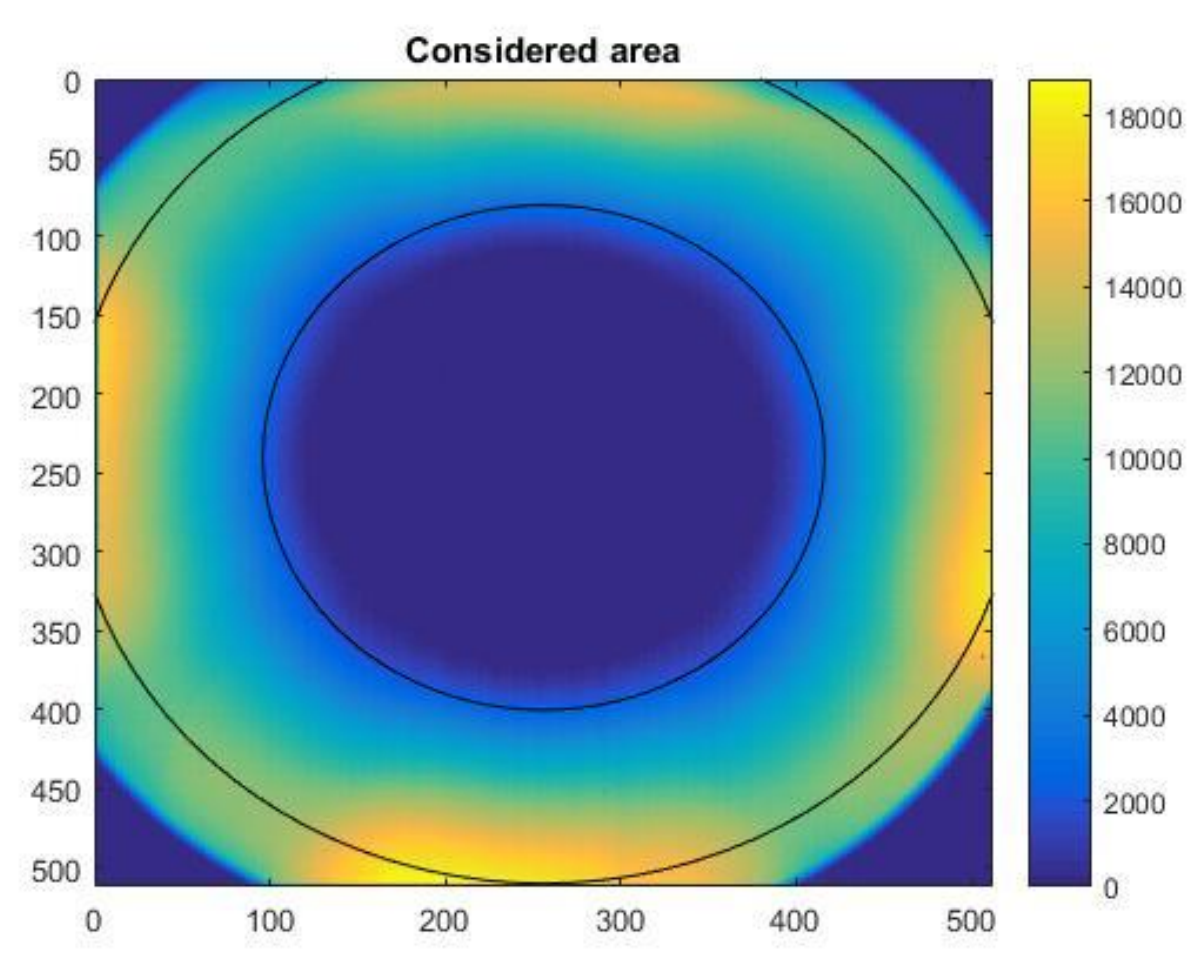




\section{Modulation Matrix Retrieval}

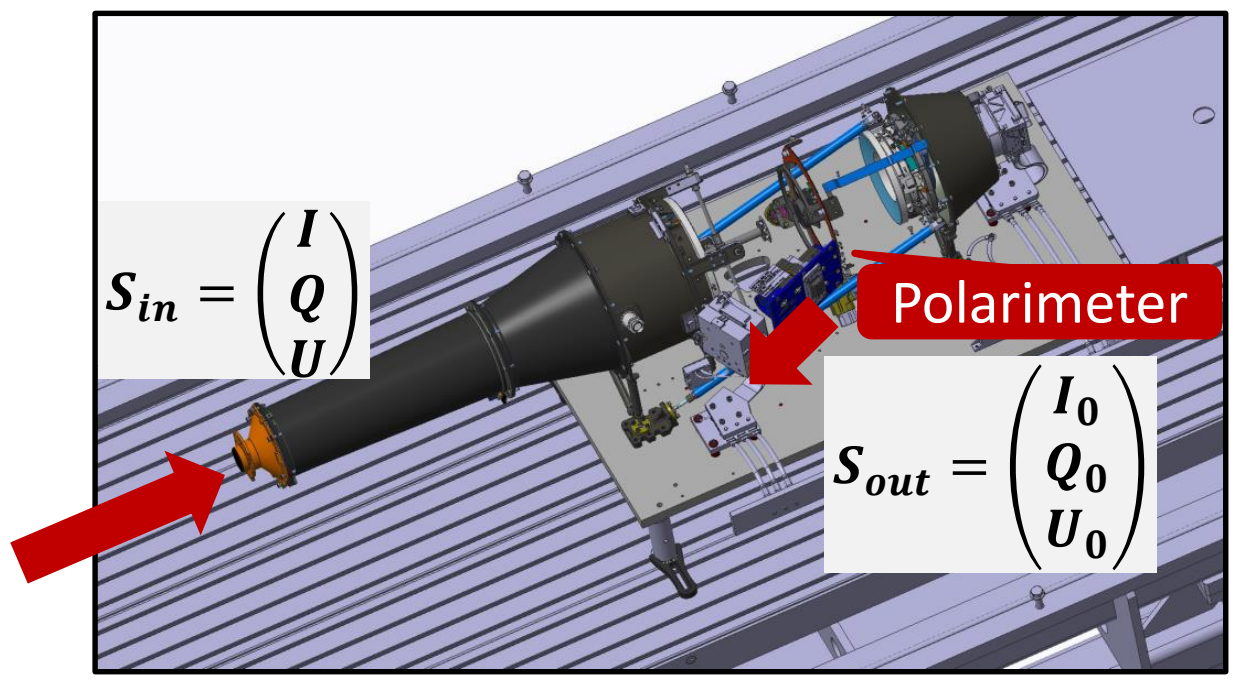

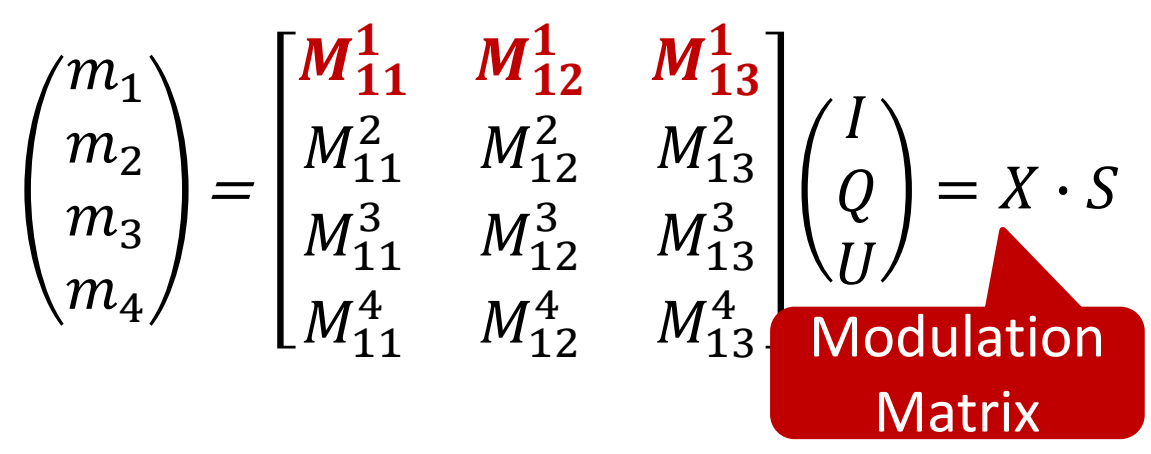

$$
S_{\text {out }}=M_{\text {polarimeter }} \cdot S_{\text {in }}
$$$$
\mathrm{M}_{\text {polarimeter }}=\left[\begin{array}{cccc}
1 & \cos \delta(V) & \sin \delta(V) & 0 \\
0 & \cos \delta(V) & \sin \delta(V) & 0 \\
0 & 0 & 0 & 0 \\
0 & 0 & 0 & 0
\end{array}\right]
$$

- Only the intensity of $S_{0}$ is measured $\rightarrow$ we are sensitive to only the first row of the polarimeter Mueller matrix.

- The Mueller matrix of the polarization modulator changes after a different value of the voltage has been applied to the LCVRs.

- For a given pre-polarizer position, we collected measurements $\left(m_{x}\right)$ related to 4 different applied voltages 


\section{Modulation Matrix Retrieval}

Considering:

- 4 voltages $\rightarrow$ Mueller matrix fixed $\rightarrow$ Modulation matrix fixed

- 4 pre-polarizer positions

Then :

it is possible to retrieve a linear system of equations for computing the modulation matrix coefficients

\begin{tabular}{|c|c|}
\hline $\begin{array}{c}\text { Pre-Pol angle in the } \\
\text { considered reference } \\
\text { frame (aligned wrt the } \\
\text { analyzer) [deg] }\end{array}$ & $\begin{array}{c}\text { Theoretical Stokes' } \\
\text { Vector }\end{array}$ \\
\hline 45 & $\left(\begin{array}{lll}1 & 0 & 1\end{array}\right)^{\top}$ \\
\hline 0 & $\left(\begin{array}{lll}1 & 1 & 0\end{array}\right)^{\top}$ \\
\hline-45 & $\left(\begin{array}{lll}1 & 0 & -1\end{array}\right)^{\top}$ \\
\hline 90 & $\left(\begin{array}{lll}1 & -1 & 0\end{array}\right)^{\top}$ \\
\hline
\end{tabular}

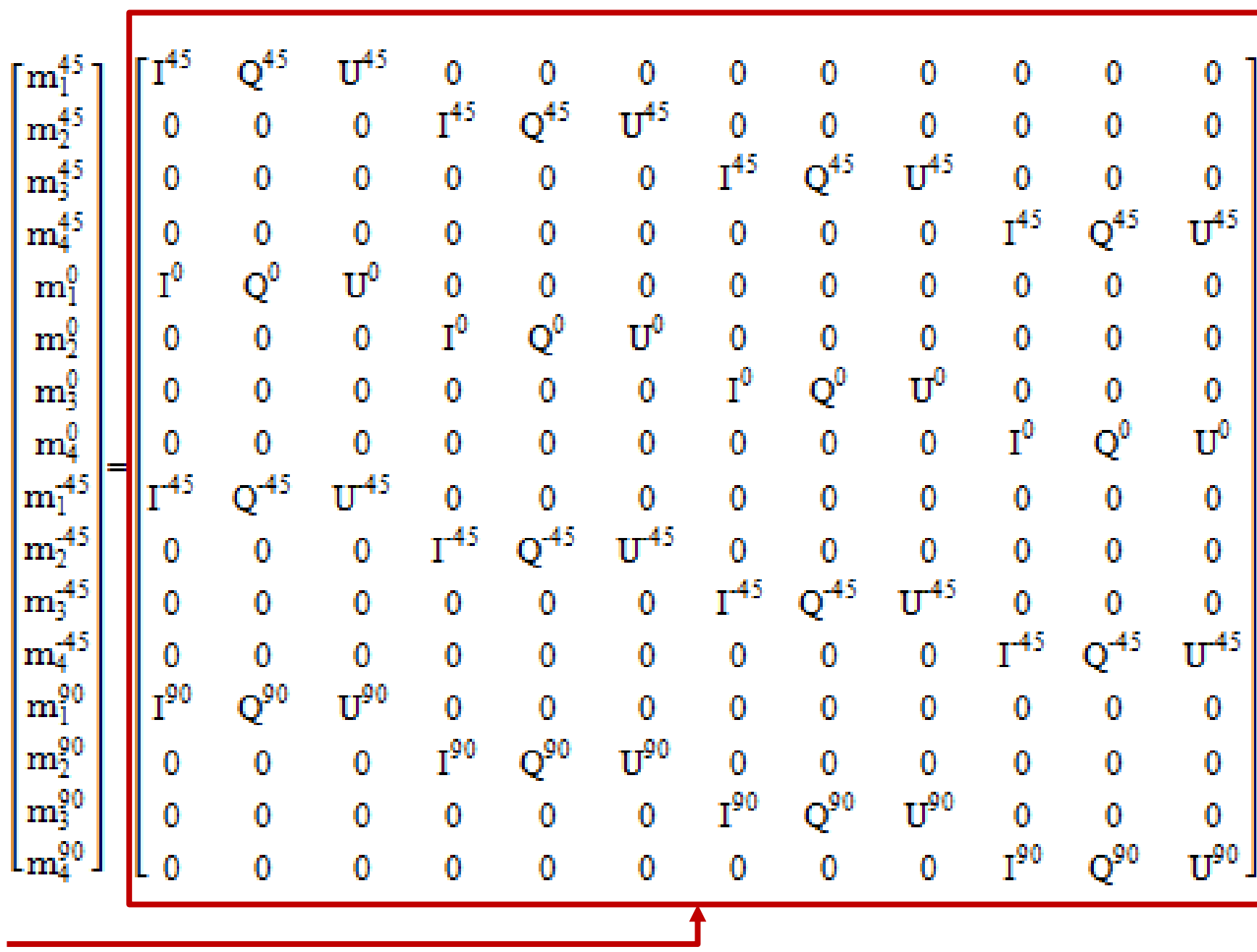

FOR EACH PIXEL 


\section{Modulation Tensor}

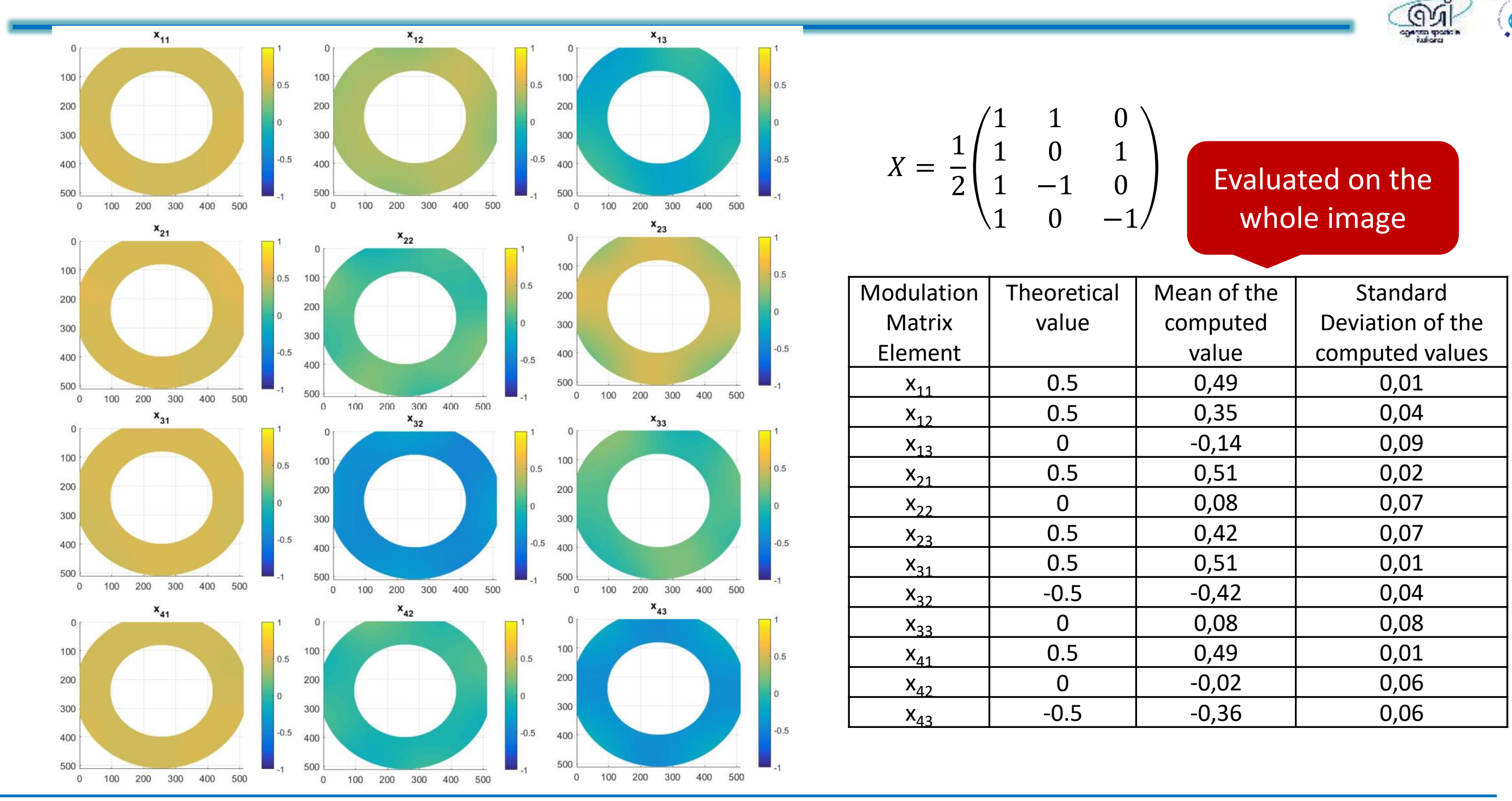




\section{Demodulation Matrix Retrieval}

The demodulation matrix must be measured, and the accuracy to which it is known will determine the accuracy of the measured Stokes parameters.

The incoming light Stokes parameters are obtained by inversion:

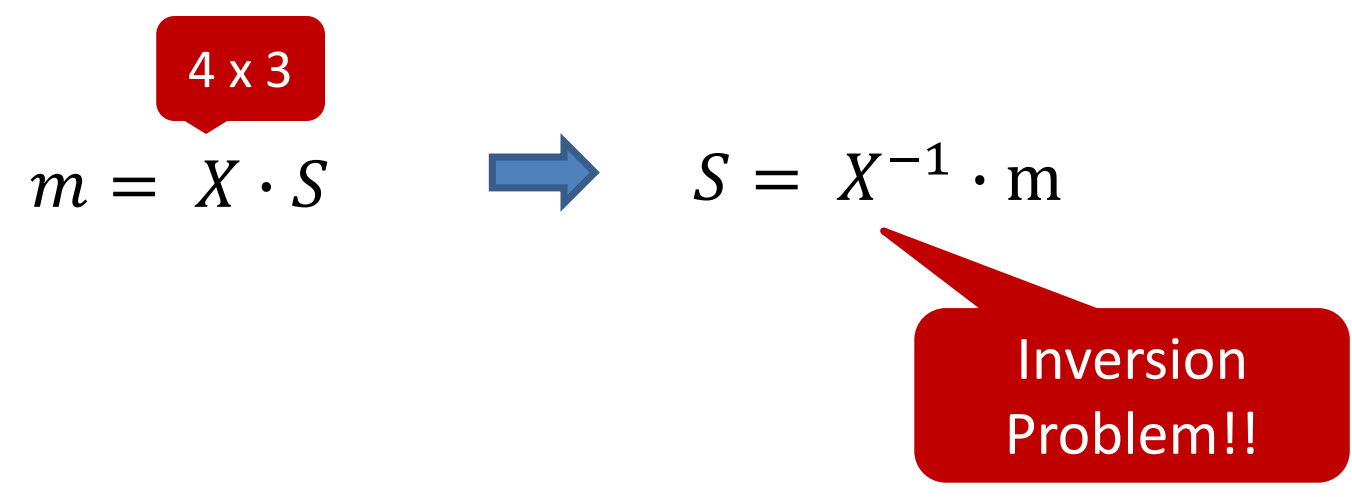

Method:

Moore-Penrose pseudoinverse $\rightarrow$ matrix that can act as a partial replacement for the matrix inverse in cases where it does not exist. This matrix is frequently used to solve a system of linear equations when the system does not have a unique solution or has many solutions. 


\section{Demodulation Tensor}

Evaluated on the whole image

\begin{tabular}{|c|c|c|c|}
\hline $\begin{array}{l}\text { Demodul } \\
\text { ation } \\
\text { Matrix } \\
\text { Element }\end{array}$ & $\begin{array}{c}\text { Theoretica } \\
\text { I value }\end{array}$ & $\begin{array}{l}\text { Mean of the } \\
\text { computed } \\
\text { value }\end{array}$ & $\begin{array}{c}\text { Std of the } \\
\text { computed } \\
\text { values }\end{array}$ \\
\hline $\mathrm{X}_{11}^{+}$ & 0.5 & 0,50 & 0,00 \\
\hline $\mathrm{x}_{12}^{+}$ & 0.5 & 0,50 & 0,00 \\
\hline $\mathrm{X}^{+}{ }_{13}$ & 0.5 & 0,50 & 0,00 \\
\hline $\mathrm{x}_{14}^{+}$ & 0.5 & 0,50 & 0,00 \\
\hline $\mathrm{x}_{21}^{+}$ & 1 & 1,12 & 0,15 \\
\hline$x_{22}^{+}$ & 0 & 0,47 & 0,38 \\
\hline $\mathrm{x}_{23}^{+}$ & -1 & $-1,39$ & 0,25 \\
\hline $\mathrm{x}_{24}^{+}$ & 0 & $-0,19$ & 0,24 \\
\hline$x_{31}^{+}$ & 0 & $-0,29$ & 0,20 \\
\hline$x_{32}^{+}$ & 1 & 1,35 & 0,34 \\
\hline $\mathrm{x}_{33}^{+}$ & 0 & 0,06 & 0,33 \\
\hline $\mathrm{X}_{34}^{+}$ & -1 & $-1,16$ & 0,31 \\
\hline
\end{tabular}


- The efficiency vector is a measure of the performance of the polarimeter.

- The Metis polarimeter is a particular case where the modulation efficiency of the circular polarization is not relevant and the maximum polarimetric efficiency for linear polarization $(Q$ and $U$ ) is required.

$$
\varepsilon_{i}=\left[\sum_{j=1}^{3}\left(X_{i j}^{-1}\right)^{2}\right]^{-1 / 2}
$$

Evaluated on the whole image
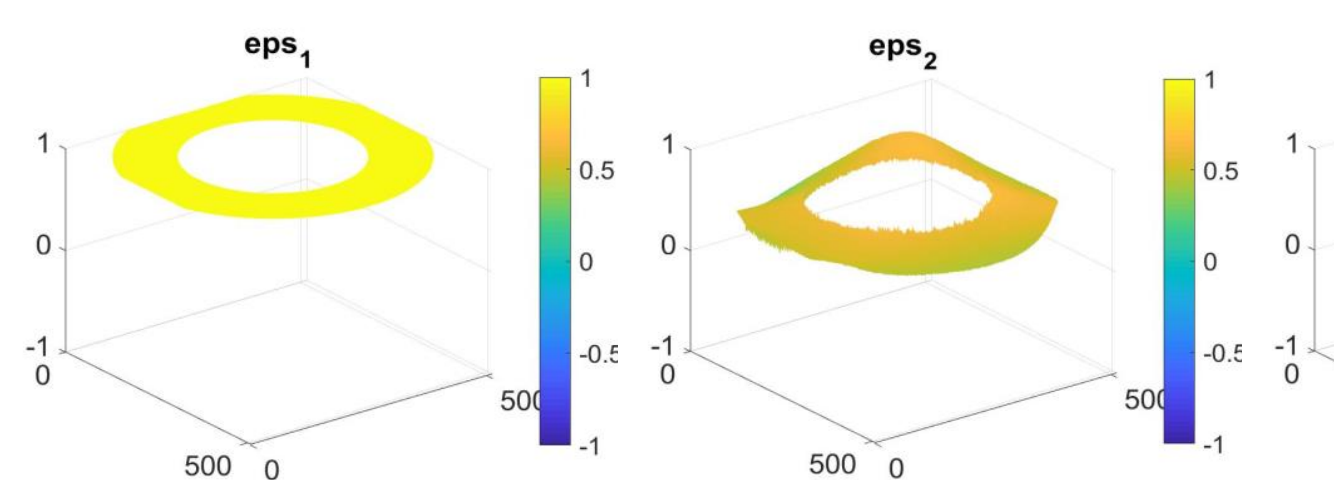

eps $_{3}$

500

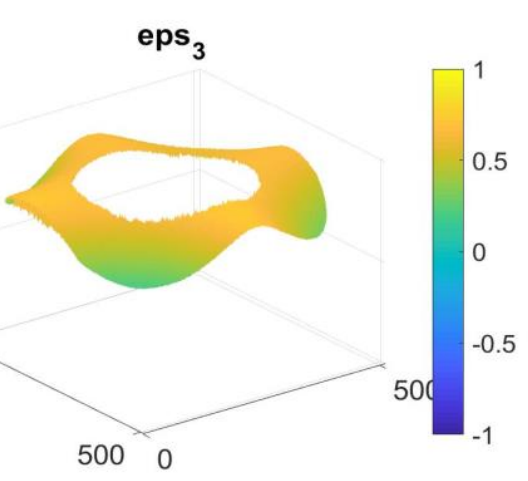

\begin{tabular}{|c|c|c|}
\hline Efficiency max & $\begin{array}{c}\text { Mean of the } \\
\text { computed value }\end{array}$ & $\begin{array}{c}\text { Standard } \\
\text { Deviation of the } \\
\text { computed values }\end{array}$ \\
\hline 1 & 1,00 & $2,05 \mathrm{e}-05$ \\
\hline $1 / \sqrt{2}$ & 0,54 & 0,07 \\
\hline $1 / \sqrt{2}$ & 0,57 & 0,11 \\
\hline
\end{tabular}




\section{Demodulation tensor verification}

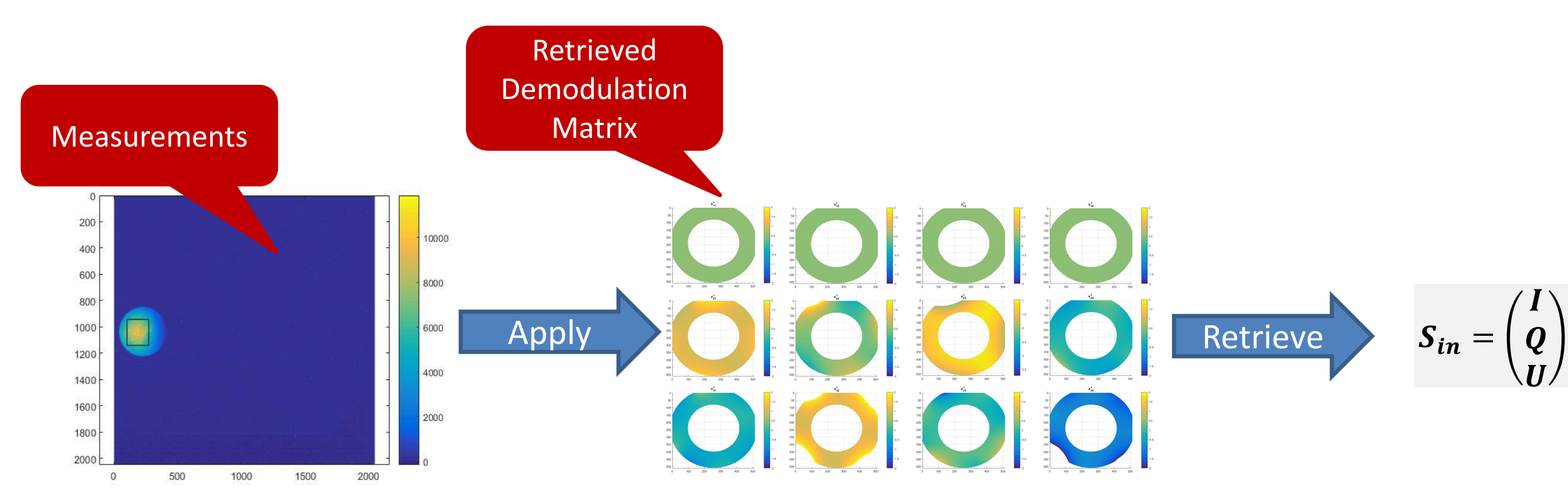

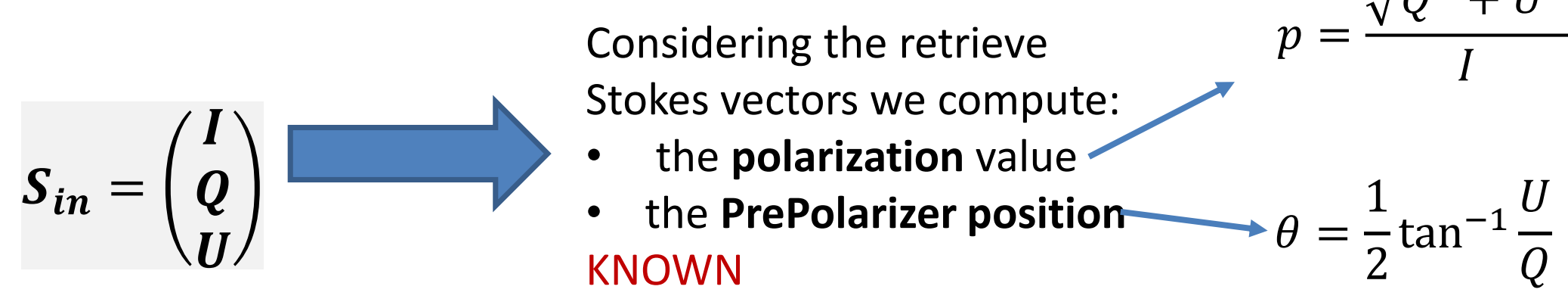




\section{Verification Results}

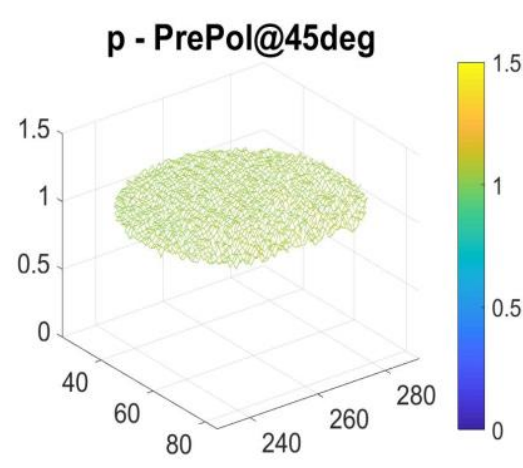

theta - PrePol@45deg

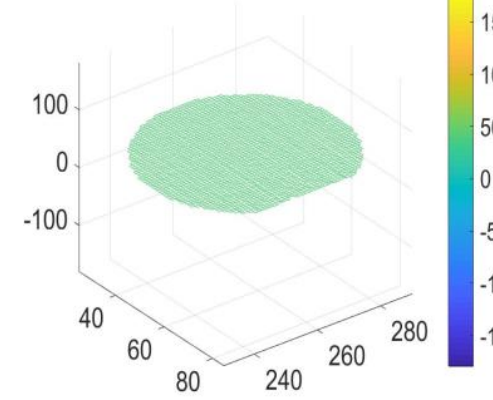

p - PrePol@0deg

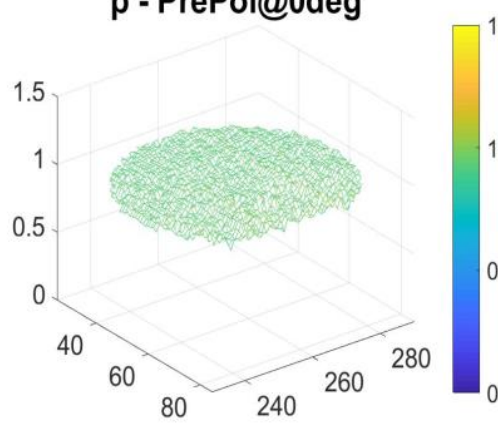

theta - PrePol@0deg

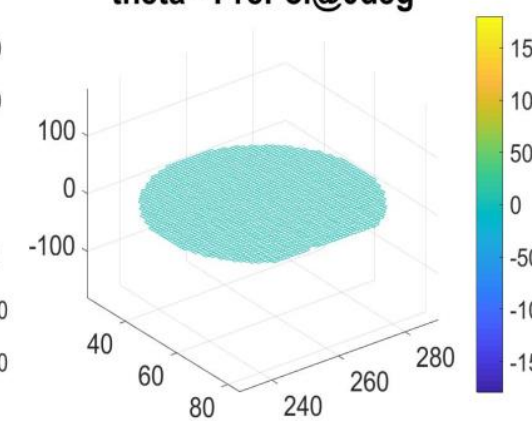

p - PrePol@-45deg

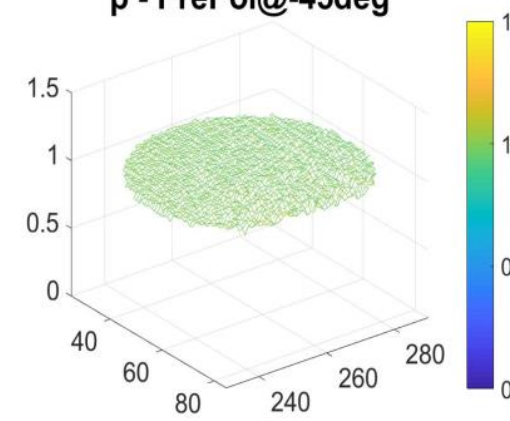

theta - PrePol@-45deg

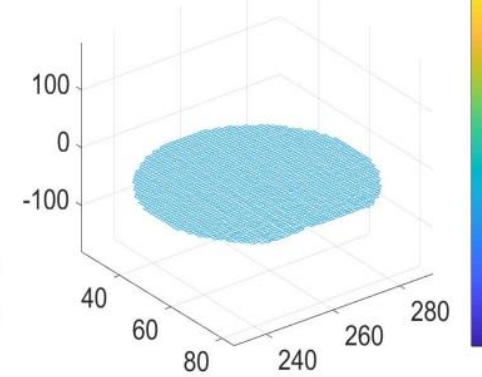

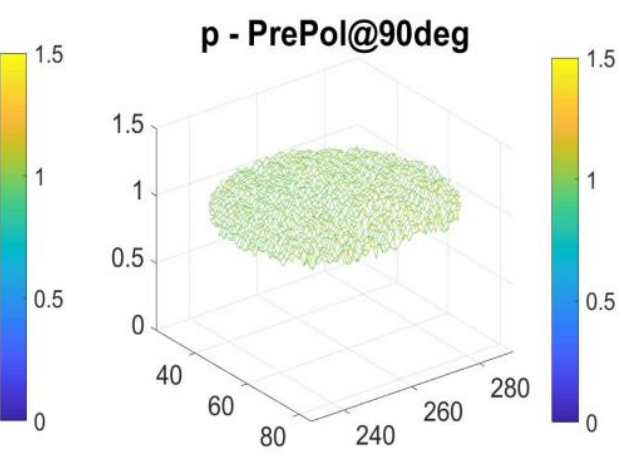

theta - PrePol@90deg

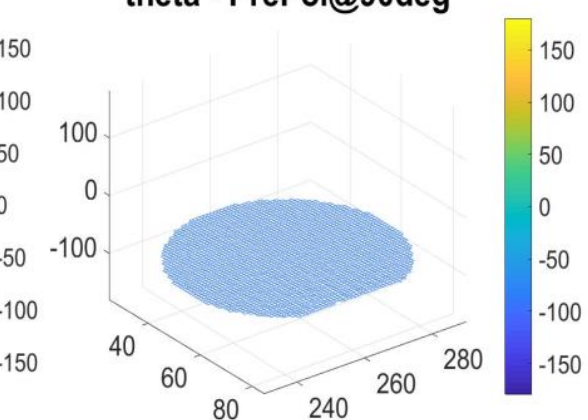

theta

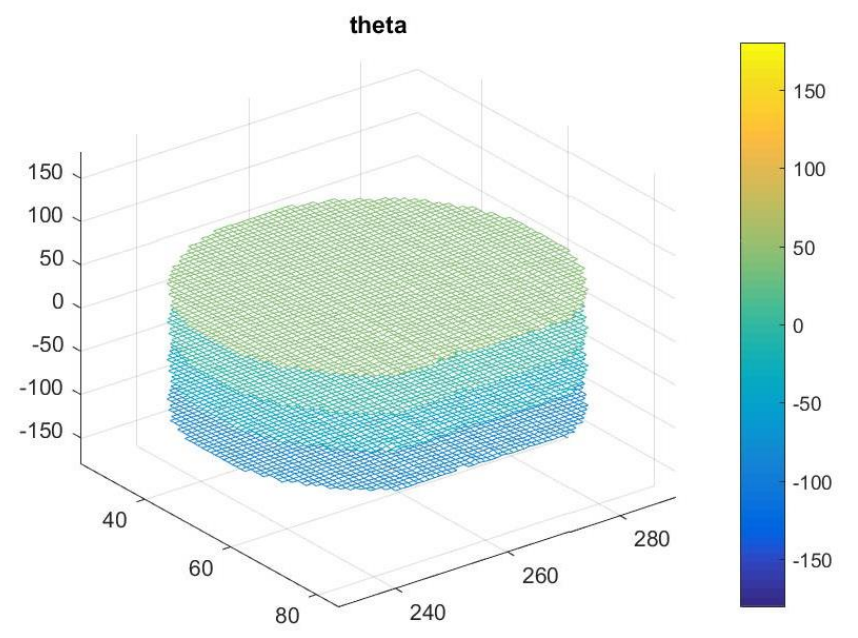

\begin{tabular}{|c|c|c|c|c|c|c|}
\hline $\begin{array}{c}\text { PrePol } \\
\text { Position [deg] }\end{array}$ & $\begin{array}{c}\mathbf{p}: \text { Theoretical } \\
\text { value }\end{array}$ & $\begin{array}{l}\text { p: Mean of the } \\
\text { computed value }\end{array}$ & $\begin{array}{l}\text { p: Standard deviation of } \\
\text { the computed value }\end{array}$ & $\begin{array}{c}\boldsymbol{\theta}: \text { Theoretical } \\
\text { value [deg] }\end{array}$ & $\begin{array}{c}\theta: \text { Mean of the } \\
\text { computed value [deg] }\end{array}$ & $\begin{array}{c}\theta: \text { Standard deviation of } \\
\text { the computed value [deg] }\end{array}$ \\
\hline 45 & 1 & 1,04 & 0,02 & 45 & 43,29 & $0,42(\sim 0,01 \mathrm{rad})$ \\
\hline 0 & 1 & 0,94 & 0,02 & 0 & $-1,04$ & $0,36(\sim 0,01$ rad $)$ \\
\hline-45 & 1 & 0,99 & 0,01 & -45 & $-44,01$ & $0,39(\sim 0,01 \mathrm{rad})$ \\
\hline 90 & 1 & 1,00 & 0,02 & 90 & 89,68 & $0,27(\sim 0,01 \mathrm{rad})$ \\
\hline
\end{tabular}




\section{Conclusions}

- The polarimetric VL channel of the Metis polarimeter has been optically characterized, by means of

- Characterization of the liquid crystals response to the applied voltage by retrieving the retardance-voltage curve;

- Characterization of the polarimetric response in visible light on the overall instrument field of view by retrieving the pixel-by-pixel demodulation matrix 


\section{Thank You for your attention}

\section{ACKNOWLEDGMENTS}

Italian Space Agency - ASI - under contract number I/013/12/0.

ALTEC Company for providing logistic and technical support.

OHB-CGS that delivered the opto-mechanical design, the electronics and the software.

Thales Alenia Space Italy was responsible for the instrument integration and alignment activity.

The MPS for providing VLDA assembly under Contract 2013-058-I.0 with the Italian Space Agency (ASI)

and the UVDA assembly thanks also to the financial support of DLR (grant 50 OT 1201). 


\section{Scientific Rationale}

Objective of the VL observations:

- to characterize the hot coronal plasma by deriving the K-corona electron density from the polarized brightness with an accuracy better than $1 \%$ in the linear polarization measurements.

An accurancy better than $1 \%$ is neded to have an error on the $\mathrm{pB}$ of about $10 \%$

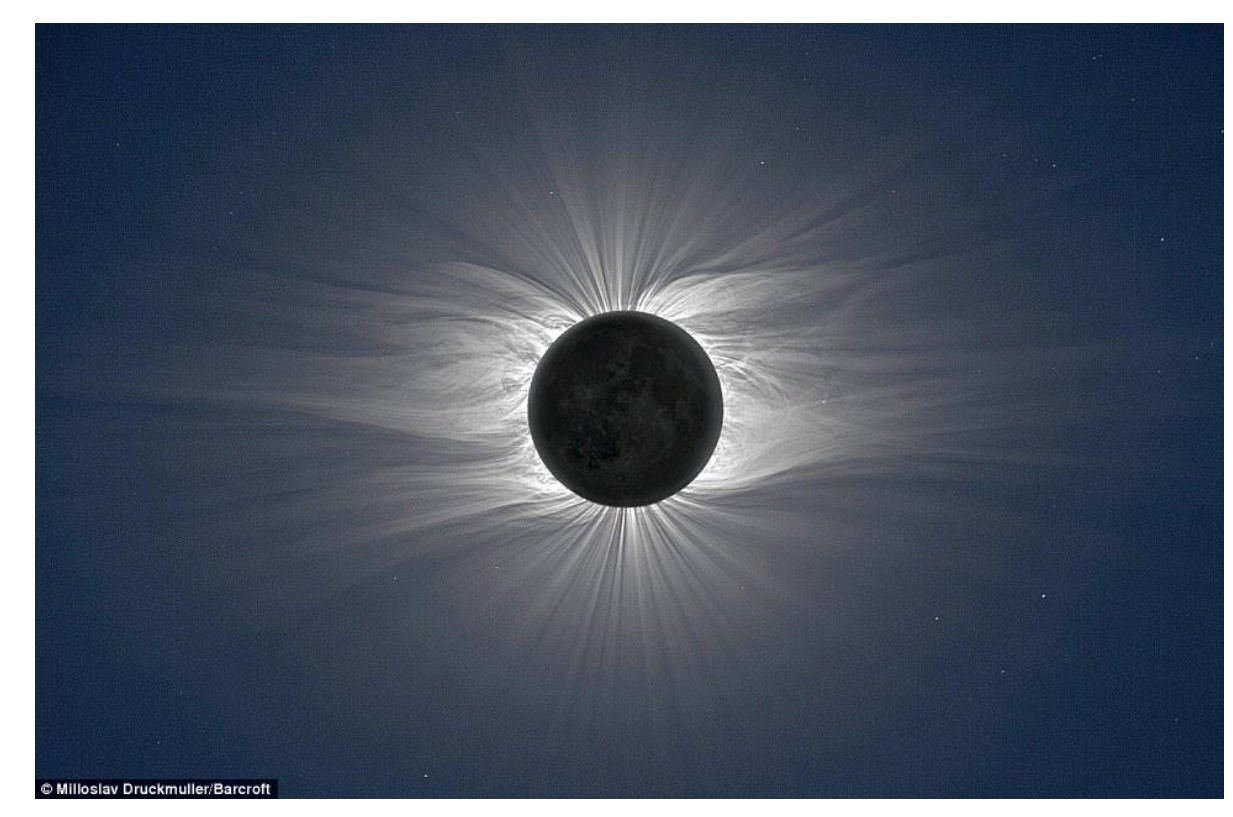

